\title{
ON DISTINGUISHED POLYNOMIALS AND THEIR PROJECTIONS
}

\author{
Erhan Çalışkan and Pilar Rueda \\ Yıldız Teknik Üniversitesi, Fen-Edebiyat Fakültesi, Matematik Bölümü \\ Davutpaşa, 34210 Esenler, Istanbul, Turkey; ercalis@yahoo.com.tr \\ Universidad de Valencia, Departamento de Análisis Matemático \\ 46.100 Burjassot, Valencia, Spain; pilar.rueda@uv.es
}

\begin{abstract}
We study projections and injections between projective tensor products spaces or spaces of polynomials and we show that the example of a polynomial constructed in [4], that is neither p-dominated nor compact, can be identified with the projection map of the symmetric tensor product onto the space. Also we give a characterization of the weak and quasi approximation properties on symmetric tensor products.
\end{abstract}

Since Ryan [17] proved that the projective symmetric $k$-fold tensor product of a Banach space $E$ is a predual of the space of continuous $k$-homogeneous polynomials on $E$; the relationship between both spaces has been deeply studied and shown to be a powerful tool in infinite dimensional holomorphy (see $[10,9,6]$ ). This duality between polynomials and linear operators on symmetric tensor product spaces is generalized to the vector-valued case by means of the process known as linearization, that arose first in [17]. The linearization process establishes an isomorphism from the space of vector valued continuous $k$-homogeneous polynomials $\mathcal{P}\left({ }^{k} E ; F\right)$ and the space of continuous linear operators defined on the projective symmetric tensor product $\hat{\otimes}_{\pi}^{k, s} E$ into $F$. Aron and Schottenloher [1] proved that the space $\mathcal{P}\left({ }^{k} E\right)$ of continuous $k$-homogeneous polynomials defined on a Banach space $E$ is complemented in $\mathcal{P}\left({ }^{n} E\right)$ whenever $k<n$. Later, Blasco [3] got the vector valued case by proving that the completed projective symmetric $k$-fold tensor product $\hat{\otimes}_{\pi}^{k, s} E$ is a complemented subspace of $\hat{\otimes}_{\pi}^{n, s} E$ for $k<n$.

In this note we undertake a detailed study of the behavior of the main distinguished polynomials and linear mappings involved in the linearization process and their interplay when the space of linear operators is identified with a complemented subspace of homogeneous polynomials. We show two applications. On the one hand we apply our study to the weak approximation property and the quasi approximation property on projective symmetric tensor products. The results we obtain are related to the results of [2], especially [2, Theorem 4.2]. On the other hand, we show that the example of a polynomial $P_{\gamma}$ constructed in [4], that is neither $p$-dominated nor compact, and weakly compact only when the underlying space is reflexive, can be identified with the projection map of the symmetric tensor product onto the space.

doi:10.5186/aasfm.2012.3745

2010 Mathematics Subject Classification: Primary 46G20; Secondary 46A32.

Key words: Projective tensor product, dominated polynomial, approximation property.

The first author was supported by TÜBİTAK - The Scientific and Technological Research Council of Turkey.

The second author was supported by Ministerio de Ciencia e Innovación MTM2011-22417. 
That allows us to understand why such a polynomial plays the role of the identity operator, and recover easily the properties concerning compactness of $P_{\gamma}$.

The paper is organized as follows. The first section is devoted to fix the notation and the basic procedures described in [1] and [3], to get complementation of the projective symmetric $n$-fold tensor product $\otimes_{s, \pi}^{n} E$ into $\otimes_{s, \pi}^{m} E$, and the space of continuous $n$-homogeneous polynomials $\mathcal{P}\left({ }^{n} E ; F\right)$ into $\mathcal{P}\left({ }^{n} E ; F\right)$, whenever $n<m$ and $E, F$ are Banach spaces. The projections considered are not canonical as they depend on a suitable continuous functional $\gamma$ in the topological dual $E^{*}$ of $E$. In Section 2 we prove, among other related results, that the injection $j^{\otimes, k}$ from $E$ into $\otimes_{s, \pi}^{k} E$ is the projection of the diagonal $k$-homogeneous polynomial $\delta_{k}: E \rightarrow \otimes_{s, \pi}^{k} E$ given by $\delta_{k}(x)=x \otimes \cdots \otimes x$. We also give the explicit description of the projection of an arbitrary continuous homogeneous polynomial in terms of its linearization. In Section 3 we apply our techniques to the study of the weak (quasi) approximation property on the completed projective symmetric tensor product $\hat{\otimes}_{s, \pi}^{k} E$. We prove that $\hat{\otimes}_{s, \pi}^{k} E$ has the weak (quasi) approximation property if and only if any compact $k$-homogeneous polynomial $P: E \rightarrow \hat{\otimes}_{s, \pi}^{k} E$ can be approximated uniformly on compact (bounded respectively) sets by finite rank polynomials. In Section 4 we prove that the projection $\pi^{\otimes, k+1}: \otimes_{s, \pi}^{k+1} E \rightarrow E$ is the linearization of the polynomial $P_{\gamma}(z):=\gamma(x)^{k} x, x \in E$, considered in [4].

\section{Notation and basic definitions}

From now on, $E$ and $F$ will denote (real or complex) Banach spaces. $\mathcal{L}(E ; F)$ denotes the space of all continuous linear maps from $E$ into $F$, endowed with the usual norm $\|T\|=\sup _{\|x\| \leq 1}\|T(x)\|, T \in \mathcal{L}(E ; F)$. The identity map on $E$ is denoted by $I d_{E}$.

Along the paper, $k$ will denote a positive integer. A map $P: E \longrightarrow F$ is said to be a continuous $k$-homogeneous polynomial if there is a continuous $k$-linear map $A: E \times \cdots \times E \longrightarrow F$ such that $P(x)=A(x, \ldots, x)$. Among all the continuous $k$ linear maps that yield the same polynomial $P$, there is only one which is symmetric and is denoted by $\stackrel{\vee}{P}$.

The set $\mathcal{P}\left({ }^{k} E ; F\right)$ of all continuous $k$-homogeneous polynomials from $E$ into $F$ form a Banach space when endowed with the norm defined as

$$
\|P\|=\sup _{\|x\| \leq 1}\|P(x)\|,
$$

for any $P \in \mathcal{P}\left({ }^{k} E ; F\right)$. We recall that a $k$-homogeneous polynomial $P: E \rightarrow F$ is said to be compact (weakly compact) if $P$ maps the closed unit ball $B_{E}$ of $E$ onto a relatively compact (respectively weakly compact) subset of $F$. The subspace of all compact $k$-homogeneous polynomials from $E$ to $F$ is denoted by $\mathcal{P}_{\mathcal{K}}\left({ }^{k} E ; F\right)$. When $k=1, \mathcal{P}_{\mathcal{K}}\left({ }^{1} E ; F\right)$ is denoted by $\mathcal{K}(E ; F)$, the space of all compact operators from $E$ to $F$. For the theory of continuous homogeneous polynomials we refer to [9].

Let $\otimes_{s, \pi}^{k} E$ denote the projective symmetric $k$-fold tensor product of $E$ and $\hat{\otimes}_{s, \pi}^{k} E$ its completion. Given $P \in \mathcal{P}\left({ }^{k} E ; F\right)$, its linearization $P_{L}$ is given by $P_{L}(x \otimes \cdots \otimes$ $x)=P(x)$ and extended by linearity and continuity. That is, if we consider the $k$ homogeneous polynomial $\delta_{k}: E \rightarrow \hat{\otimes}_{s, \pi}^{k} E$ defined by $\delta_{k}(x)=x \otimes \cdots \otimes x=: \otimes_{k} x$, then 
$P_{L}$ is the unique continuous linear operator from $\hat{\otimes}_{s, \pi}^{k} E$ to $F$ such that $P=P_{L} \circ \delta_{k}$. Notice that $\delta_{1}$ is the identity on $E$.

Blasco [3] proved that $\mathcal{P}\left({ }^{k} E ; F\right)$ is a complemented subspace of $\mathcal{P}\left({ }^{m} E ; F\right)$, for any $k<m$. The scalar case was proved first by Aron and Schottenloher [1]. Let us outline the vector valued analogue of the projection defined there. Following the ideas in [1], choose $e \in E$ and $\gamma \in E^{*}$ such that $\|e\|=\gamma(e)=\|\gamma\|=1$. Define $j_{k, F}: \mathcal{P}\left({ }^{k} E ; F\right) \rightarrow \mathcal{P}\left({ }^{k+1} E ; F\right)$ by

$$
j_{k, F}(Q)(x)=\gamma(x) Q(x),
$$

for each $Q \in \mathcal{P}\left({ }^{k} E ; F\right)$ and $x \in E$. It is clear that $j_{k, F}$ is injective. Indeed, if $Q$ is a non zero polynomial which is continuous, we can find an open ball $B$ where $Q$ does not take the value 0 ; as $\|\gamma\|=1, \gamma$ cannot be identically zero on $B$. Define $\pi_{k, F}: \mathcal{P}\left({ }^{k+1} E ; F\right) \rightarrow \mathcal{P}\left({ }^{k+1} E ; F\right)$ by

$$
\pi_{k, F}(P)(x)=P(x)-P(x-\gamma(x) e),
$$

for each $P \in \mathcal{P}\left({ }^{k+1} E ; F\right)$ and $x \in E$. An easy calculation shows that $\pi_{k, F}^{2}=\pi_{k, F}$ and as in [1] both $\pi_{k, F}$ and $j_{k, F}$ have the same image. Indeed,

$$
\left(j_{k, F}\right)^{-1} \circ \pi_{k, F} \circ j_{k, F}=I d_{\mathcal{P}\left({ }^{k} E ; F\right)}
$$

and if $P \in \mathcal{P}\left({ }^{k+1} E ; F\right)$ then $\pi_{k, F}(P)=j_{k, F}\left(Q_{P}\right)$, where $Q_{P}$ is given by

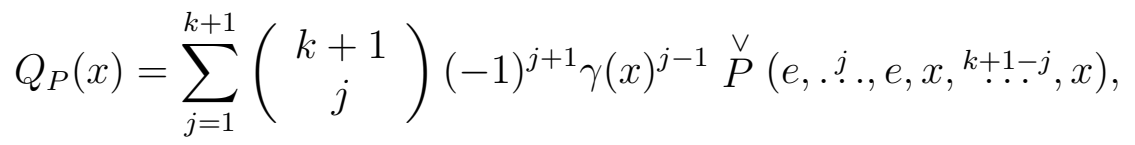

for all $x \in E$. Then, $\pi_{k, F}\left(\mathcal{P}\left({ }^{k+1} E ; F\right)\right)$ is isomorphic to $\mathcal{P}\left({ }^{k} E ; F\right)$. Hence, the space $\mathcal{P}\left({ }^{k} E ; F\right)$ is isomorphic to the complemented subspace $j_{k, F}\left(\mathcal{P}\left({ }^{k} E ; F\right)\right)$ of $\mathcal{P}\left({ }^{k+1} E ; F\right)$, whose projection is $\pi_{k, F}$.

Let us define

$$
q_{k, F}:=\left(j_{k, F}\right)^{-1} \circ \pi_{k, F}: \mathcal{P}\left({ }^{k+1} E ; F\right) \rightarrow \mathcal{P}\left({ }^{k} E ; F\right) .
$$

Following [3], for each positive integer $k$ there is an isomorphism into, that we denote by $j_{k}^{\otimes}: \otimes_{s, \pi}^{k} E \rightarrow \otimes_{s, \pi}^{k+1} E$ such that

$$
j_{k}^{\otimes}\left(\delta_{k}(x)\right) \gamma(x)=\delta_{k+1}(x)-\delta_{k+1}(x-\gamma(x) e)
$$

for all $x \in E$. Moreover, the linear operator $\pi_{k}^{\otimes}: \otimes_{s, \pi}^{k+1} E \rightarrow \otimes_{s, \pi}^{k} E$ given by $\pi_{k}^{\otimes}\left(\otimes_{k+1} x\right)=\gamma(x) \otimes_{k} x$ is a projection onto. It is proved in [3] that

$$
\pi_{k}^{\otimes} \circ j_{k}^{\otimes}=I d_{\otimes_{s, \pi}^{k} E}
$$

is the identity map on $\otimes_{s, \pi}^{k} E$.

Finally let us define $\pi^{\otimes, m+1}: \otimes_{s, \pi}^{m+1} E \rightarrow E$ by $\pi^{\otimes, m+1}=\pi_{1}^{\otimes} \circ \cdots \circ \pi_{m}^{\otimes}$.

\section{Projections of polynomials}

We define $j^{\otimes, k}: E \rightarrow \otimes_{s, \pi}^{k} E$ given by $j^{\otimes, k}:=j_{k-1}^{\otimes} \circ \cdots \circ j_{1}^{\otimes}$. So defined, the map $j^{\otimes, k}$ is an isomorphism into. Let $j_{F}^{k}: \mathcal{L}(E ; F) \rightarrow \mathcal{P}\left({ }^{k} E ; F\right)$ be the injection defined by $j_{F}^{k}:=j_{k-1, F} \circ \cdots \circ j_{1, F}$, and let $q_{F}^{k}: \mathcal{P}\left({ }^{k} E ; F\right) \rightarrow \mathcal{L}(E ; F)$ be given by $q_{F}^{k}:=q_{1, F} \circ \cdots \circ q_{k-1, F}$. 
For short, we will denote $E_{k}:=\otimes_{s, \pi}^{k} E$ whenever it appears as a subindex. For instance,

$$
\begin{aligned}
\pi_{k-1, E_{k+1}} & =\pi_{k-1, \otimes_{s, \pi}^{k+1} E}: \mathcal{P}\left({ }^{k} E ; \otimes_{s, \pi}^{k+1} E\right) \rightarrow \mathcal{P}\left({ }^{k} E ; \otimes_{s, \pi}^{k+1} E\right), \\
q_{E_{k}}^{k} & =q_{\otimes_{s, \pi}^{k} E}^{k}: \mathcal{P}\left({ }^{k} E ; \otimes_{s, \pi}^{k} E\right) \rightarrow \mathcal{L}\left(E ; \otimes_{s, \pi}^{k} E\right)
\end{aligned}
$$

and

$$
j_{E_{k}}^{k}=j_{\otimes_{s, \pi}^{k} E}^{k}: \mathcal{L}\left(E ; \otimes_{s, \pi}^{k} E\right) \rightarrow \mathcal{P}\left({ }^{k} E ; \otimes_{s, \pi}^{k} E\right) .
$$

Lemma 1. For each positive integer $k, \pi_{k-1, E_{k+1}}\left(j_{k}^{\otimes} \circ \delta_{k}\right)=j_{k}^{\otimes} \circ\left(\pi_{k-1, E_{k}}\left(\delta_{k}\right)\right)$.

Proof. Notice that $j_{k}^{\otimes} \circ \delta_{k} \in \mathcal{P}\left({ }^{k} E ; \otimes_{s, \pi}^{k+1} E\right)$. Let $x \in E$. Then

$$
\begin{aligned}
\pi_{k-1, E_{k+1}}\left(j_{k}^{\otimes} \circ \delta_{k}\right)(x) & =j_{k}^{\otimes}\left(\delta_{k}(x)\right)-j_{k}^{\otimes}\left(\delta_{k}(x-\gamma(x) e)\right) \\
& =j_{k}^{\otimes}\left(\delta_{k}(x)-\delta_{k}(x-\gamma(x) e)\right)=j_{k}^{\otimes}\left(\pi_{k-1, E_{k}}\left(\delta_{k}\right)(x)\right) .
\end{aligned}
$$

Proposition 2. Let $k$ be a positive integer, $k \geq 2$. Then

(i) $\pi_{k-1, E_{k}}\left(\delta_{k}\right)=j_{k-1, E_{k}}\left(j_{k-1}^{\otimes} \circ \delta_{k-1}\right)$, and

(ii) $q_{k-1, E_{k}}\left(\delta_{k}\right)=j_{k-1}^{\otimes} \circ \delta_{k-1}$.

Proof. (i) For each $x \in E$, using (2) in the second equality, we get

$$
\begin{aligned}
\pi_{k-1, E_{k}}\left(\delta_{k}\right)(x) & =\delta_{k}(x)-\delta_{k}(x-\gamma(x) e)=j_{k-1}^{\otimes}\left(\delta_{k-1}(x)\right) \gamma(x) \\
& =j_{k-1, E_{k}}\left(j_{k-1}^{\otimes} \circ \delta_{k-1}\right)(x) .
\end{aligned}
$$

(ii) Using (i) in the second equality we get

$$
\begin{aligned}
q_{k-1, E_{k}}\left(\delta_{k}\right) & =\left(j_{k-1, E k}\right)^{-1} \circ \pi_{k-1, E_{k}}\left(\delta_{k}\right) \\
& =\left(j_{k-1, E k}\right)^{-1}\left(j_{k-1, E k}\left(j_{k-1}^{\otimes} \circ \delta_{k-1}\right)\right)=j_{k-1}^{\otimes} \circ \delta_{k-1} .
\end{aligned}
$$

Theorem 3. For each positive integer $k, q_{E_{k}}^{k}\left(\delta_{k}\right)=j^{\otimes, k}$.

Proof. Using Proposition 2(ii) in the second equality we get

$$
\begin{aligned}
q_{E_{k}}^{k}\left(\delta_{k}\right) & =q_{1, E_{k}} \circ \cdots \circ q_{k-1, E_{k}}\left(\delta_{k}\right)=q_{1, E_{k}} \circ \cdots \circ q_{k-2, E_{k}}\left(j_{k-1}^{\otimes} \circ \delta_{k-1}\right) \\
& =q_{1, E_{k}} \circ \cdots \circ\left(j_{k-2, E_{k}}\right)^{-1} \circ \pi_{k-2, E_{k}}\left(j_{k-1}^{\otimes} \circ \delta_{k-1}\right) .
\end{aligned}
$$

So, from Lemma 1 it follows that

$$
\begin{aligned}
q_{E_{k}}^{k}\left(\delta_{k}\right) & =q_{1, E_{k}} \circ \cdots \circ\left(j_{k-2, E_{k}}\right)^{-1}\left(j_{k-1}^{\otimes} \circ\left(\pi_{k-2, E_{k-1}}\left(\delta_{k-1}\right)\right)\right) \\
& =q_{1, E_{k}} \circ \cdots \circ\left(j_{k-2, E_{k}}\right)^{-1}\left(x \mapsto j_{k-1}^{\otimes}\left(\pi_{k-2, E_{k-1}}\left(\delta_{k-1}\right)(x)\right)\right) \\
& =q_{1, E_{k}} \circ \cdots \circ\left(j_{k-2, E_{k}}\right)^{-1}\left(x \mapsto j_{k-1}^{\otimes}\left(j_{k-2}^{\otimes}\left(\delta_{k-2}(x)\right) \gamma(x)\right)\right) \\
& =q_{1, E_{k}} \circ \cdots \circ\left(j_{k-2, E_{k}}\right)^{-1}\left(x \mapsto j_{k-1}^{\otimes}\left(j_{k-2}^{\otimes}\left(\delta_{k-2}(x)\right)\right) \gamma(x)\right) \\
& =q_{1, E_{k}} \circ \cdots \circ\left(j_{k-2, E_{k}}\right)^{-1}\left(j_{k-2, E_{k}}\left(j_{k-1}^{\otimes} \circ j_{k-2}^{\otimes} \circ \delta_{k-2}\right)\right. \\
& =q_{1, E_{k}} \circ \cdots \circ q_{k-3, E_{k}}\left(j_{k-1}^{\otimes} \circ j_{k-2}^{\otimes} \circ \delta_{k-2}\right)=\cdots \\
& =j_{k-1}^{\otimes} \circ \cdots \circ j_{1}^{\otimes} \circ \delta_{1}=j_{k-1}^{\otimes} \circ \cdots \circ j_{1}^{\otimes}=j^{\otimes, k} .
\end{aligned}
$$

So far we have studied how projections and injections between projective tensor products spaces or spaces of polynomials affect to the polynomial $\delta_{k}$. We are now in conditions to study general $k$-homogeneous polynomials $P$. 
Theorem 4. Let $k$ be a positive integer, $k \geq 2$, and let $P \in \mathcal{P}\left({ }^{k} E ; F\right)$. Then $\pi_{k-1, F}(P)=P_{L} \circ\left(\pi_{k-1, E_{k}}\left(\delta_{k}\right)\right)=P_{L} \circ\left(j_{k-1, E_{k}}\left(j_{k-1}^{\otimes} \circ \delta_{k-1}\right)\right)=j_{k, F}\left(P_{L} \circ\left(q_{k-1, E_{k}}\left(\delta_{k}\right)\right)\right)$.

Proof. Let $x \in E$. Then

$$
\begin{aligned}
\pi_{k-1, F}(P)(x) & =P(x)-P(x-\gamma(x) e)=P_{L}\left(\delta_{k}(x)-\delta_{k}(x-\gamma(x) e)\right) \\
& =P_{L}\left(\pi_{k-1, E_{k}}\left(\delta_{k}\right)(x)\right)=P_{L} \circ\left(j_{k-1, E_{k}}\left(j_{k-1}^{\otimes} \circ \delta_{k-1}\right)\right)(x) .
\end{aligned}
$$

On the other hand, using Proposition 2(ii) in the second equality and (2) in the third one,

$$
\begin{aligned}
j_{k, F}\left(P_{L} \circ\left(q_{k-1, E_{k}}\left(\delta_{k}\right)\right)\right)(x) & =P_{L} \circ\left(q_{k-1, E_{k}}\left(\delta_{k}\right)\right)(x) \gamma(x)=P_{L}\left(j_{k-1}^{\otimes} \circ \delta_{k-1}(x) \gamma(x)\right) \\
& =P_{L}\left(\delta_{k}(x)-\delta_{k}(x-\gamma(x) e)\right) \\
& =P(x)-P(x-\gamma(x) e)=\pi_{k-1, F}(P)(x) .
\end{aligned}
$$

\section{The weak approximation property and the quasi approximation property for the space $\hat{\otimes}_{s, \pi}^{k} E$}

We say that $E$ has the weak approximation property (in short, WAP) if given a compact operator $T \in \mathcal{L}(E ; E)$, a compact set $K \subset E$ and $\epsilon>0$, there is a finite rank operator $T_{f} \in \mathcal{L}(E ; E)$ such that $\sup _{x \in K}\left\|T x-T_{f} x\right\| \leq \epsilon$. We say that $E$ has the quasi approximation property (in short, QAP) if given a compact operator $T \in \mathcal{L}(E ; E)$ and $\epsilon>0$, there is a finite rank operator $T_{f} \in \mathcal{L}(E ; E)$ such that $\left\|T-T_{f}\right\| \leq \epsilon$. Lindenstrauss and Tzafriri [13, p. 37, Problem 1.e.9] posed the following problem that remains open:

Does the QAP imply the approximation property on Banach spaces?

Trying to get a solution to that problem, Choi and Kim [7] introduced the weak approximation property and characterized separable reflexive Banach spaces having the QAP in terms of a stronger condition than the WAP. For examples of Banach spaces having the $W A P$ and the $Q A P$ and failing to have the $W A P$ and the $Q A P$ see [7, Examples 2.1 and 2.3]. Obviously, the QAP implies the WAP but we know no examples of Banach spaces which have the WAP but fails to have the QAP. For further study and relations between the $W A P$, the $Q A P$ and other variants of the approximation property we refer to $[8,11,12]$. In this section we will give a characterization of the spaces $\hat{\otimes}_{s, \pi}^{k} E$ having the WAP and the QAP.

Before giving the results about the WAP and the QAP, let us give a lemma concerning the projection of polynomials, which we use in the subsequent proofs. For each $y \in F$ and $P \in \mathcal{P}\left({ }^{k} E\right)$ define $(P \otimes y)(x)=P(x) y, x \in E$. So defined, $P \otimes y \in \mathcal{P}\left({ }^{k} E ; F\right)$ and its image is contained in the 1-dimensional subspace of $F$ generated by $y$. A polynomial $P \in \mathcal{P}\left({ }^{k} E ; F\right)$ is said to be of finite rank if there are $n \in \mathbf{N}, P_{1}, \ldots, P_{n} \in \mathcal{P}\left({ }^{k} E\right)$ and $y_{1}, \ldots, y_{n} \in F$ such that $P=\sum_{j=1}^{n} P_{j} \otimes y_{j}$. Let $\mathcal{P}_{\mathcal{F}}\left({ }^{k} E ; F\right)$ denote the space of all finite rank $k$-homogeneous polynomials from $E$ to $F$. When $k=1, \mathcal{P}_{\mathcal{F}}\left({ }^{1} E ; F\right)$ is simply denoted by $\mathcal{F}(E ; F)$, the space of all finite rank operators from $E$ to $F$.

Lemma 5. For each $k \in \mathbf{N}$,

(i) $j_{k, F}\left(\mathcal{P}_{\mathcal{F}}\left({ }^{k} E ; F\right)\right) \subset \mathcal{P}_{\mathcal{F}}\left({ }^{k+1} E ; F\right)$,

(ii) $\pi_{k, F}\left(\mathcal{P}_{\mathcal{F}}\left({ }^{k+1} E ; F\right)\right) \subset \mathcal{P}_{\mathcal{F}}\left({ }^{k+1} E ; F\right)$,

(iii) $q_{k, F}\left(\mathcal{P}_{\mathcal{F}}\left({ }^{k+1} E ; F\right)\right) \subset \mathcal{P}_{\mathcal{F}}\left({ }^{k} E ; F\right)$, and 
(iv) $q_{F}^{k}\left(\mathcal{P}_{\mathcal{F}}\left({ }^{k} E ; F\right)\right) \subset \mathcal{F}(E ; F)$.

Proof. (i) Let $Q \in \mathcal{P}_{\mathcal{F}}\left({ }^{k} E ; F\right)$. Then we can write $Q=\sum_{j=1}^{n} Q_{j} \otimes y_{j}$ for some $n \in \mathbf{N}, Q_{1}, \ldots, Q_{n} \in \mathcal{P}\left({ }^{k} E\right)$ and $y_{1}, \ldots, y_{n} \in F$. Hence, for all $x \in E$

$$
j_{k, F}(Q)(x)=\sum_{j=1}^{n} j_{k, F}\left(Q_{j} \otimes y_{j}\right)(x)=\sum_{j=1}^{n} \gamma(x) Q_{j}(x) y_{j}=\sum_{j=1}^{n} j_{k, \mathbf{C}}\left(Q_{j}\right)(x) y_{j} .
$$

Then $j_{k, F}(Q)$ belongs to $\mathcal{P}_{\mathcal{F}}\left({ }^{k+1} E ; F\right)$.

(ii) If $P=\sum_{j=1}^{l} P_{j} \otimes z_{j} \in \mathcal{P}_{\mathcal{F}}\left({ }^{k+1} E ; F\right)$, then $\pi_{k, F}\left(P_{j} \otimes z_{j}\right)(x)=P_{j}(x) z_{j}-P_{j}(x-\gamma(x) e) z_{j}=\pi_{k, \mathbf{C}}\left(P_{j}\right)(x) z_{j}=\left(\pi_{k, \mathbf{C}}\left(P_{j}\right) \otimes z_{j}\right)(x)$. Hence, $\pi_{k, F}(P)=\sum_{j=1}^{l} \pi_{k, \mathbf{C}}\left(P_{j}\right) \otimes z_{j} \in \mathcal{P}_{\mathcal{F}}\left({ }^{k+1} E ; F\right)$.

(iii) Recall that $q_{k, F}=\left(j_{k, F}\right)^{-1} \circ \pi_{k, F}$. Part (iii) follows from (ii) and the fact that if $P \in \mathcal{P}_{\mathcal{F}}\left({ }^{k+1} E ; F\right)$ then $Q_{P}$ defined as in (1) belongs to $\mathcal{P}_{\mathcal{F}}\left({ }^{k} E ; F\right)$. Indeed, if we write $P=\sum_{i=1}^{n} P_{i} \otimes y_{i}$, for some $P_{1}, \ldots, P_{n} \in \mathcal{P}\left({ }^{k+1} E\right)$ and $y_{1}, \ldots, y_{n} \in F$, then

$$
\pi_{k, F}\left(P_{i} \otimes y_{i}\right)=\pi_{k, \mathbf{C}}\left(P_{i}\right) \otimes y_{i}=j_{k, \mathbf{C}}\left(Q_{\pi_{k, \mathbf{C}}\left(P_{i}\right)}\right) \otimes y_{i}=j_{k, F}\left(Q_{\pi_{k, \mathbf{C}}\left(P_{i}\right)} \otimes y_{i}\right) .
$$

Hence, for every $i=1, \ldots, n$

$$
Q_{P \otimes y_{i}}=Q_{\pi_{k, \mathbf{C}}(P)} \otimes y_{i} .
$$

(iv) follows from (iii).

Lemma 6. Let $k$ be a positive integer, $k \geq 2$. If $T \in \mathcal{K}(E ; F)$ then $j_{F}^{k}(T) \in$ $\mathcal{P}_{\mathcal{K}}\left({ }^{k} E ; F\right)$.

Proof. Define the $k$-homogeneous polynomial $P_{\gamma}: E \rightarrow E$ given by $P_{\gamma}(x):=$ $\gamma(x)^{k-1} x$. Then

$$
j_{F}^{k}(T)=j_{k-1, F} \circ \cdots \circ j_{1, F}(T)=\gamma^{k-1} \cdot T=T \circ P_{\gamma} .
$$

Since $P_{\gamma}$ is a continuous $k$-homogeneous polynomial, it maps bounded sets into bounded sets. The compactness of $T$ and (3) yields the result.

The polynomial $P_{\gamma}$ will be studied in Section 4 . Note that the result is valid if we replace $T$ with a compact $k$-homogeneous polynomial and $j_{F}^{k}$ with $j_{k, F}$.

In the next proposition we give a characterization of the WAP on symmetric projective tensor products. Let $\tau_{0}$ denote the topology on $\mathcal{P}\left({ }^{k} E ; F\right)$ of uniform convergence on compact subsets of $E$.

Proposition 7. Let $E$ be a Banach space. Consider the following conditions:

(1) $\hat{\otimes}_{s, \pi}^{k} E$ has the WAP.

(2) $\mathcal{P}_{\mathcal{K}}\left({ }^{k} E ; \hat{\otimes}_{s, \pi}^{k} E\right)={\overline{\mathcal{P}_{\mathcal{F}}\left({ }^{k} E ; \hat{\otimes}_{s, \pi}^{k} E\right)}}^{\tau_{0}}$.

(3) $\mathcal{K}\left(E ; \hat{\otimes}_{s, \pi}^{k} E\right)={\overline{\mathcal{F}}\left(E ; \hat{\otimes}_{s, \pi}^{k} E\right)}^{\tau_{0}}$.

Then 1 and 2 are equivalent and they imply 3.

Proof. $1 \Rightarrow 2$ : Let $P \in \mathcal{P}_{\mathcal{K}}\left({ }^{k} E ; \hat{\otimes}_{s, \pi}^{k} E\right), K$ compact in $E$ and $\epsilon>0$. Since $P_{L} \in \mathcal{K}\left(\hat{\otimes}_{s, \pi}^{k} E ; \hat{\otimes}_{s, \pi}^{k} E\right)$ due to [17] and $\delta_{k}(K)$ is compact in $\hat{\otimes}_{s, \pi}^{k} E$, there exists $T \in \mathcal{F}\left(\hat{\otimes}_{s, \pi}^{k} E ; \hat{\otimes}_{s, \pi}^{k} E\right)$ such that $\left\|P_{L}-T\right\|_{\delta_{k}(K)}<\epsilon$. Let $Q \in \mathcal{P}\left({ }^{k} E ; \hat{\otimes}_{s, \pi}^{k} E\right)$ whose 
linearization is $T$. Since $T$ has finite rank then $Q$ also has finite rank [16]. If $x \in K$, then

$$
\pi(P(x)-Q(x))=\pi\left(P_{L}\left(\delta_{k}(x)\right)-T\left(\delta_{k}(x)\right)\right) \leq\left\|P_{L}-T\right\|_{\delta_{k}(K)}<\epsilon,
$$

and this proves 2 .

$2 \Rightarrow 3$ : For short, let $j:=j_{\hat{\otimes}_{s, \pi}^{k} E}^{k}$ and $q:=q_{\hat{\otimes}_{s, \pi}^{k} E}^{k}$. Let $T \in \mathcal{K}\left(E ; \hat{\otimes}_{s, \pi}^{k} E\right), K$ a compact subset of $E$ and $\epsilon>0$. By Lemma $6, j(T) \in \mathcal{P}_{\mathcal{K}}\left({ }^{k} E ; \hat{\otimes}_{s, \pi}^{k} E\right)$. Then from 2 applied to $j(T)$, there exists $P \in \mathcal{P}_{\mathcal{F}}\left({ }^{k} E ; \hat{\otimes}_{s, \pi}^{k} E\right)$ such that $\|j(T)-P\|_{K}<\epsilon$. By Lemma $5, q(P) \in \mathcal{F}\left(E ; \hat{\otimes}_{s, \pi}^{k} E\right)$. Since

$$
\|T-q(P)\|_{K}=\|q \circ j(T)-q(P)\|_{K} \leq\|q\|\|j(T)-P\|_{K}<\epsilon\|q\|,
$$

part 3 follows.

$2 \Rightarrow 1$ : Take $T \in \mathcal{K}\left(\hat{\otimes}_{s, \pi}^{k} E ; \hat{\otimes}_{s, \pi}^{k} E\right)$ and let $K$ be a compact subset of $\hat{\otimes}_{s, \pi}^{k} E$ and $\epsilon>0$. As in the projective tensor product, the compact set $K$ is contained in the closed convex hull $\bar{\Gamma}\left(\delta_{k}\left(K_{1}\right)\right)$ of $\delta_{k}\left(K_{1}\right)$ for some compact $K_{1} \subset E$. Let $P:=T \circ \delta_{k} \in$ $\mathcal{P}\left({ }^{k} E ; \hat{\otimes}_{s, \pi}^{k} E\right)$. Since $P_{L}=T$ is compact, then $P$ is also compact [17]. By 2 there exists $Q \in \mathcal{P}_{\mathcal{F}}\left({ }^{k} E ; \hat{\otimes}_{s, \pi}^{k} E\right)$ such that $\|P-Q\|_{K_{1}}<\epsilon$. Then $Q_{L} \in \mathcal{F}\left(\hat{\otimes}_{s, \pi}^{k} E ; \hat{\otimes}_{s, \pi}^{k} E\right)$ and

$$
\left\|T-Q_{L}\right\|_{K} \leq\left\|T-Q_{L}\right\|_{\bar{\Gamma}\left(\delta\left(K_{1}\right)\right)}=\left\|T-Q_{L}\right\|_{\delta\left(K_{1}\right)}=\|P-Q\|_{K_{1}}<\epsilon,
$$

and 1 is proved.

A similar characterization can be proved for the QAP. We include the proof for the sake of completeness.

Proposition 8. Let $E$ be a Banach space. Consider the following conditions:

(1) $\hat{\otimes}_{s, \pi}^{k} E$ has the $Q A P$

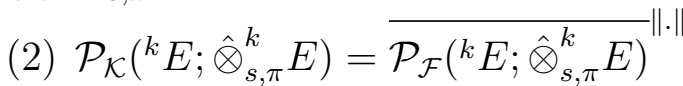

(3) $\mathcal{K}\left(E ; \hat{\otimes}_{s, \pi}^{k} E\right)=\overline{\mathcal{F}\left(E ; \hat{\otimes}_{s, \pi}^{k} E\right)}\|\cdot\|$

Then 1 and 2 are equivalent and they imply 3.

Proof. $1 \Rightarrow 2$ : Let $P \in \mathcal{P}_{\mathcal{K}}\left({ }^{k} E ; \hat{\otimes}_{s, \pi}^{k} E\right)$ and $\epsilon>0$. Since $P_{L} \in \mathcal{K}\left(\hat{\otimes}_{s, \pi}^{k} E ; \hat{\otimes}_{s, \pi}^{k} E\right)$ and $\bar{\Gamma}\{\delta(x):\|x\|<1\}$ is the closed unit ball of $\hat{\otimes}_{s, \pi}^{k} E$ (see for example [16, Remark 2.5(c)]), there exists $T \in \mathcal{F}\left(\hat{\otimes}_{s, \pi}^{k} E ; \hat{\otimes}_{s, \pi}^{k} E\right)$ such that $\left\|P_{L}-T\right\|_{\bar{\Gamma}\left(\delta_{k}\left(U_{E}\right)\right)}<\epsilon$, where $U_{E}$ denotes the open unit ball of $E$. Let $Q \in \mathcal{P}\left({ }^{k} E ; \hat{\otimes}_{s, \pi}^{k} E\right)$ whose linearization is $T$. Since $T$ has finite rank then $Q$ also has finite rank [16]. If $x \in U_{E}$ then

$$
\begin{aligned}
\pi(P(x)-Q(x)) & =\pi\left(P_{L}\left(\delta_{k}(x)\right)-T\left(\delta_{k}(x)\right)\right) \leq\left\|P_{L}-T\right\|_{\delta_{k}\left(U_{E}\right)} \\
& \leq\left\|P_{L}-T\right\|_{\bar{\Gamma}\left(\delta_{k}\left(U_{E}\right)\right)}<\epsilon,
\end{aligned}
$$

hence, by continuity, for any $x \in B_{E}$ we get that $\pi(P(x)-Q(x)) \leq \epsilon$, which shows 2 .

$2 \Rightarrow 3$ : Let $T \in \mathcal{K}\left(E ; \hat{\otimes}_{s, \pi}^{k} E\right)$ and $\epsilon>0$. Since $j(T) \in \mathcal{P}_{\mathcal{K}}\left({ }^{k} E ; \hat{\otimes}_{s, \pi}^{k} E\right)$, by 2 applied to $j(T)$, there exists $P \in \mathcal{P}_{\mathcal{F}}\left({ }^{k} E ; \hat{\otimes}_{s, \pi}^{k} E\right)$ such that $\|j(T)-P\|<\epsilon$. By Lemma $5, q(P) \in \mathcal{F}\left(E ; \hat{\otimes}_{s, \pi}^{k} E\right)$. Since

$$
\|T-q(P)\|=\|q \circ j(T)-q(P)\| \leq\|q\|\|j(T)-P\|<\epsilon\|q\|,
$$


we have 3 .

$2 \Rightarrow 1$ : Take $T \in \mathcal{K}\left(\hat{\otimes}_{s, \pi}^{k} E ; \hat{\otimes}_{s, \pi}^{k} E\right)$ and let $\epsilon>0$. We know that $B_{\hat{\otimes}_{s, \pi}^{k} E}=$ $\bar{\Gamma}\left(\delta\left(U_{E}\right)\right)$. Let $P:=T \circ \delta \in \mathcal{P}\left({ }^{k} E ; \hat{\otimes}_{s, \pi}^{k} E\right)$. Since $P_{L}=T$ is compact, then $P$ is also compact [17]. By 2 there exists $Q \in \mathcal{P}_{\mathcal{F}}\left({ }^{k} E ; \hat{\otimes}_{s, \pi}^{k} E\right)$ such that $\|P-Q\|<\epsilon$. Then $Q_{L} \in \mathcal{F}\left(\hat{\otimes}_{s, \pi}^{k} E ; \hat{\otimes}_{s, \pi}^{k} E\right)$ and $\left\|T-Q_{L}\right\|_{\bar{\Gamma}\left(\delta\left(U_{E}\right)\right)}=\left\|T-Q_{L}\right\|_{\delta\left(U_{E}\right)}=\left\|T \circ \delta-Q_{L} \circ \delta\right\|_{U_{E}}=\|P-Q\|_{U_{E}}=\|P-Q\|<\epsilon$, which shows 1 .

Since $E$ is a complemented subspace of the space $\hat{\otimes}_{s, \pi}^{k} E$ it is easy to see that $E$ has the WAP (resp., the QAP) whenever $\hat{\otimes}_{s, \pi}^{k} E$ has the WAP (resp., the QAP). We do not know if the reverse implication is true.

\section{Connection with $p$-dominated polynomials}

A continuous $n$-homogeneous polynomial $P: E \longrightarrow F$ is said to be $p$-dominated if there is a constant $C>0$ such that

$$
\left(\sum_{j=1}^{m}\left\|P\left(x_{j}\right)\right\|^{p / n}\right)^{n / p} \leq C \sup _{x^{*} \in B_{E^{*}}}\left(\sum_{j=1}^{m}\left|\left\langle x^{*}, x_{j}\right\rangle\right|^{p}\right)^{n / p}
$$

for any positive integer $m$ and any finite sequence of vectors $x_{1}, \ldots, x_{m} \in E$. The least constant $C$ for which the above inequality holds is denoted by $\|P\|_{d, p}$. Let $\mathcal{P}_{d, p}\left({ }^{n} E ; F\right)$ denote the set of all $p$-dominated $n$-homogeneous polynomials from $E$ into $F$. It is well known that $\mathcal{P}_{d, p}\left({ }^{n} E ; F\right)$ endowed with \|\|$_{d, p}$ is a Banach space when $p \geq n$ and a $p / n$-norm when $p<n$ (see, e.g., [5]).

Dominated polynomials appeared as a generalization of summing linear operators to the wider context of homogeneous polynomials. Actually, $p$-dominated 1homogeneous polynomials are just $p$-summing linear operators.

Let $E$ be an infinite dimensional Banach space, let $n$ be a positive integer and choose $\varphi \in E^{\prime}, \varphi \neq 0$. Botelho [4] constructed an $n$-homogeneous polynomial $P_{\varphi}: E \rightarrow E$ which plays the role of the identity operator in the sense that (i) $P_{\varphi}$ is neither $p$-dominated nor compact, (ii) $P_{\varphi}$ is weakly compact if and only if $E$ is reflexive. The polynomial $P_{\varphi}$ is given by $P_{\varphi}(x):=\varphi(x)^{n-1} x, x \in E$. Part (i) was actually proved by Matos [15]. Next proposition clarifies the inherent nature of $P_{\varphi}$ and shows that (ii) should not surprise us at the same time that provides a straightforward proof.

Proposition 9. The projection $\pi^{\otimes, n+1}$ is the linearization of $P_{\gamma}$.

Proof. Let $x \in E$. From the definition of $\pi^{\otimes, n+1}$ it follows that

$$
\pi^{\otimes, n+1} \circ \delta_{n+1}(x)=\pi^{\otimes, n+1}(x \otimes \cdots \otimes x)=\gamma(x)^{n} x .
$$

To be more precise, the linearization of $P_{\gamma}$ is the extension $\hat{\pi}^{\otimes, n+1}$ of $\pi^{\otimes, n+1}$ to the complete projective symmetric tensor product $\hat{\otimes}_{s, \pi}^{n+1} E$. Ryan [17] proved that an $n$ homogeneous polynomial is compact (weakly compact) if and only if its linearization is compact (respectively weakly compact). Applying these results to the polynomial $P_{\gamma}$ we get that $P_{\gamma}$ is weakly compact if and only if $\hat{\pi}^{\otimes, n+1}$ is weakly compact. By the Open Mapping Theorem, $\hat{\pi}^{\otimes, n+1}$ is an open mapping and so, $\hat{\pi}^{\otimes, n+1}\left(B_{\hat{\otimes}_{s, \pi}^{n+1} E}\right)$ contains 
a ball, which is weakly relatively compact in $E$ whenever $P_{\gamma}$ is weakly compact. Then we have got (ii).

Acknowledgements. This paper was partly written while the first author was visiting the Department of Mathematical Sciences at Kent State University. He thanks this Department for its kind hospitality. The authors thank the referee for his careful reading and comments that have improved the paper.

\section{References}

[1] Aron, R. M., and M. Schottenloher: Compact holomorphic mappings on Banach spaces and the approximation property. - J. Funct. Anal. 21:1, 1976, 7-30.

[2] Berrios, S., and G. Botelho: Approximation properties determined by operator ideals and approximability of homogeneous polynomials and holomorphic functions. - Studia Math. 208, 2012, 97-116.

[3] Blasco, F.: Complementation in spaces of symmetric tensor products and polynomials. Studia Math. 123:2, 1997, 165-173.

[4] Botelho, G.: Weakly compact and absolutely summing polynomials. - J. Math. Anal. Appl. 265:2, 2002, 458-462.

[5] Botelho, G.: Ideals of polynomials generated by weakly compact operators. - Note Mat. 25, 2005, 69-102.

[6] Botelho, G., D. Pellegrino, and P. Rueda: Pietsch's factorization theorem for dominated polynomials. - J. Funct. Anal. 243, 2007, 257-269.

[7] Chor, C., and J. M. KIm: Weak and quasi approximations properties in Banach spaces. - J. Math. Anal. Appl. 316, 2006, 722-735.

[8] Choi, C., J. M. Kim, and K. Y. LEE: Right and left weak approximation properties in Banach spaces. - Canad. Math. Bull. 52, 2009, 28-38.

[9] Dineen, S.: Complex analysis on infinite dimensional spaces. - Springer-Verlag, London, 1999.

[10] Floret, K.: Natural norms on symmetric tensor products of normed spaces. - Note Mat. 17, 1997, 153-188.

[11] Kim, J. M.: Dual problems for weak and quasi approximation properties. - J. Math. Anal. Appl. 321, 2006, 569-575.

[12] Kim, J. M.: On relations between weak approximation properties and their inheritances to subspaces. - J. Math. Anal. Appl. 324, 2006, 721-727.

[13] Lindenstrauss, J., and L. Tzafriri: Classical Banach spaces I. Sequence spaces. - Springer, Berlin, 1977.

[14] Matos, M.: Absolutely summing holomorphic mappings. - An. Acad. Brasil. Ciênc. 68, 1996, $1-13$.

[15] Matos, M.: Nonlinear absolutely summing mappings. - Math. Nachr. 258, 2003, 71-89.

[16] MujICA, J.: Linearization of bounded holomorphic mappings on Banach spaces. - Trans. Amer. Math. Soc. 324, 1991, 867-887.

[17] RyAn, R.: Applications of topological tensor products to infinite dimensional holomorphy. Ph. D. Thesis, Trinity College, Dublin, 1980. 